\title{
EL PERFIL PROFESIONAL DE LA CLASE POLÍTICA LOCAL DE SAN LUIS POTOSÍ, MÉXICO
}

Miguel Ángel Vega Campos ${ }^{1}$

Resumen. En términos de Gaetano Mosca (1972), en cualquier sociedad existen dos clases de personas, una de los gobernantes y la otra de los gobernados. La clase de los gobernantes a la que Mosca denomina como la clase política, es la que realiza todas las funciones políticas, monopoliza el poder y goza de las ventajas que ello trae consigo. La elaboración del trabajo surgió de la inquietud por conocer cuál es el perfil político y administrativo de la clase política que accedió al poder local en el Estado de San Luis Potosí, a través de los procesos electorales celebrados durante los años 2006, 2009 y 2012, para ocupar los cargos de diputados locales y presidentes municipales. La ejecución del estudio se apoyó en información biográfica publicada por el Consejo Estatal Electoral y de Participación Ciudadana de San Luis Potosí (CEEPAC), de los servidores públicos electos en los referidos procesos políticos. El estudio pretende generar información que concientice a la ciudadanía en general, a las autoridades electorales y a los propios partidos políticos, respecto a la pertinencia de cuidar el perfil profesional de los candidatos a ocupar puestos decisores en el sector público, cuya intervención impacta directa o indirectamente también en el ámbito privado. En la medida que esta situación prevalezca, se continuará padeciendo de una clase política subpreparada e incapaz de cambiar el rumbo de la gestión pública. La profesionalización del sector público es conveniente no solamente en el nivel técnico o instrumental, sino también en los puestos de elección popular, también llamados puestos políticos.

Palabras clave: Clase política, procesos políticos, perfil profesional, elecciones.

Abstract. In terms of Gaetano Mosca (1972), there are two groups of people in any society: governing ones and governed. The governing ones, whom Mosca denominates the political class, are the people who execute all of the political functions, monopolize power and leverage the advantages those circumstances bring along. The elaboration of this research

\footnotetext{
${ }^{1}$ Doctor en administración por la Universidad Autónoma de San Luis Potosí (UASLP). Actualmente se desempeña como profesor investigador y Coordinador de la Maestría en Administración con Énfasis en Gestión Pública, en la Facultad de Contaduría y Administración de la UASLP. Correo electrónico: vegacamposnet@hotmail.com
} 
originated from the inquisitiveness to know the political and administrative profile of the political class that acceded to local power in the State of San Luis Potosí, through the elections held during years 2006, 2009 and 2012, in order to occupy the position of deputies and mayors. The realization of this research is supported in biographical information, published by the Electoral and Civic Participation State Council of San Luis Potosí (CEEPAC), of the elect public servants in the aforementioned political processes. The research expects to generate information which in turn raises awareness on citizens, electoral authorities and political parties themselves, regarding the appropriateness to care for the professional profile of the candidates employed in decision-making positions on public sector, whose intervention impacts, directly or indirectly, the private sector. As far as this situation prevails, the ailment of an underprepared political class incapable to change the path of public management will continue to exist. The professionalization of the public sector is convenient not only at a technical or instrumental level but also in popular election positions called political positions.

Key words: Political class, political processes, professional profile, elections. 


\section{Introducción}

No obstante que el artículo 35 de la Constitución Política de los Estados Unidos Mexicanos (CPEUM), estipula en su fracción II que es un derecho de los ciudadanos mexicanos poder ser votados para todos los cargos de elección popular, teniendo las calidades que establezca la ley aplicable; también debe destacarse que lo deseable sería que al campo público pudieran llegar los mejores hombres y mujeres, aquellos que cuenten con los conocimientos, las habilidades, las capacidades, las aptitudes y las actitudes necesarias para ocupar alguno de los cargos a los que se refiere la CPEUM. Es decir, que reúnan el perfil profesional adecuado para que su desempeño gubernamental sea efectivo y acorde a las necesidades que demandan las condiciones actuales.

Con relación al párrafo que antecede, para el caso de San Luis Potosí, el artículo $7^{\circ}$ de la Constitución Política local (CPSLP), señala que "en el Estado de San Luis Potosí la protección de los derechos de sus habitantes y la permanente búsqueda del interés público son la base y objeto de las instituciones políticas y sociales". Como se puede observar, tanto las instituciones políticas como las sociales, deben pugnar por lograr condiciones que permitan alcanzar mejores niveles de bienestar para los habitantes de la entidad federativa a la que aquí se hace referencia.

En términos de lo expuesto en renglones anteriores, los partidos políticos se constituyen como un mecanismo importante que puede influir favorablemente, para que a través de éstos se canalicen hacia el sector público, los hombres y las mujeres que cuenten con el perfil profesional idóneo para mejorar las condiciones que actualmente prevalecen en todos los ámbitos de gobierno, ya sea del orden federal, estatal o municipal. 
Ante este panorama, surgen inquietudes como: ¿realmente los partidos políticos están canalizando a los hombres y mujeres que cuentan con los mejores perfiles profesionales, de acuerdo a los cargos para los que los postulan?, ¿qué partido político es el que más se preocupa por esta situación?, ¿qué situación prevalece de un proceso electoral a otro?, ¿qué perfil político o administrativo es el que prevalece?, entre otras interrogantes.

A fin de encontrar respuestas a los cuestionamientos antes referidos, se tomó la decisión de investigar cuál es el perfil profesional que prevalece en la llamada clase política local del Estado de San Luis Potosí, la cual accedió al poder a través de los procesos electorales realizados en los años 2006, 2009 y 2012. Es importante señalar que para efectos de este trabajo, solamente se consideraron los puestos electos de diputados y presidentes municipales. Se trata de un estudio preponderantemente descriptivo y de corte transversal, apoyado en los datos biográficos de los personajes antes aludidos, los cuales fueron publicados por el CEEPAC.

\section{Marco conceptual ¿Élite o clase política?}

Dentro de la literatura sobre el tema, se dice que el primer científico social en hablar acerca de las "élites", o mejor dicho de las "clases políticas", como él prefería llamarlas, fue Gaetano Mosca (Blacha, 2005). En este sentido, Mosca (1972, p. 1) señala que: Entre las tendencias y hechos constantes que se encuentran en todos los organismos políticos, uno es tan obvio que es obvio a toda manifestación: en todas las sociedades, comenzando desde mediocremente desarrolladas y que apenas han arribado a lo primordial de la civilización, terminando por las más numerosas y más cultas, existen dos clases de personas, una de los gobernantes y la otra de los gobernados. La primera, que es siempre la menos numerosa, realiza todas las funciones políticas, monopoliza el poder y goza de las ventajas que ello trae consigo; mientras que la segunda, más numerosa, es dirigida y regulada por la primera, de un modo más o menos legal, ya más o menos arbitrario y violento, y ella la provee, al menos aparentemente, de los medios materiales de subsistencia y de aquellos que para la vitalidad del organismo político son necesarios. 
Pareto es uno de los autores que difundió ampliamente el concepto de "élite" y que es un término mediante el cual los franceses designaron a "los mejores" (Blacha, 2005). Conforme el tiempo ha transcurrido, el término élite ha dejado de ser una palabra francesa para designar a "quienes, por cualquier razón -quizá muy poco verdadera- se destacan y se sitúan por encima de los demás", donde nada tiene que ver una superioridad moral (Meisel, 1975; citado por Blacha, 2005). En términos de Wright Mills (Sin fecha; citado por Blacha, 2005), el estudio de las "élites" resulte conveniente, en virtud de su centralidad en la comprensión de la estructura social que habitamos.

Por lo que hace al concepto de "clase política", Bell (Meisel, 1975; citado por Blacha, 2005) la conceptualiza como "un grupo poseedor del poder, con una comunidad establecida de intereses y una continuidad de intereses". Sigue exponiendo Meisel (1975; citado por Blacha, 2005) que "la clase política es un hecho y una necesidad a la vez, ya que sin ella no se podría gobernar la sociedad". Es así que "la clase política justifica su posición mediante 'principios abstractos' o una 'fórmula' que es compartida y aceptada por la masa poco educada; que refleja su carácter y funciones" (Blacha, 2005).

En palabras de Hurtado (1996, pp. 105-106), "élite política designa al extracto superior de actores políticos que detentan cuotas significativas de poder político, ocupan posiciones relevantes dentro de la estructura formal de poder o gobierno, y/o participan formal o informalmente en la toma de decisiones". Para efectos de este documento, se utilizará el concepto de clase política expresado por Hurtado (1996, p. 106), al señalar que por clase política se entiende "el conjunto de individuos que estando fuera del gobierno o dentro de éste ocupan posiciones o cumplen funciones significativas dentro de un sistema político", 
como fue el caso de los servidores públicos (diputados locales y presidentes municipales) que accedieron al poder a través de los procesos políticos de los años 2006, 2009 y 2012 en el Estado de San Luis Potosí.

\section{La profesionalización del gobierno}

Por lo que respecta a la profesionalización del gobierno, vale la pena mencionar lo que expone Mesta (2004; citado por Vega, 2016, p. 117) cuando indica que "el aparato administrativo en México no ha tenido la capacidad de responder eficazmente a las demandas de la sociedad, causando en nuestro país, como en casi todas las naciones del mundo, el cuestionamiento del Estado obeso, ineficaz, ineficiente e improductivo"; de ahí que en verdad se requiere dirigir los pasos hacia una verdadera profesionalización gubernamental.

En cuanto al tema de la profesionalización, Mejía (2004; citado por Vega, 2016, p. 117) señala que "la profesionalización en la administración pública responde a una necesidad de disponer de especialistas en todos los campos, tanto técnicas científicas como políticas en el campo gubernamental, favoreciendo los mejores resultados”. Pero para que esto suceda, es requisito indispensable que los sistemas de personal público se rediseñen y se adecuen a las exigencias de los nuevos tiempos (Pérez, 2004).

Por otro lado, Uvalle (2004; citado por Vega, 2016, p. 117) señala que "la profesionalización del servicio público es un imperativos para que los asuntos y los problemas públicos sean solucionados por personal apto y responsable ante las instituciones de la democracia". Sigue exponiendo el mismo autor, que "la profesionalización es antídoto para erradicar las prácticas de improvisación, ineficiencia y clientelismo, y de ese modo, evitar señales confiables al público ciudadano, los mercados y las organizaciones de la sociedad en el sentido de que la certidumbre institucional de los servidores públicos para dar cauce a la carrera administrativa, 
es garantía para que la administración pública quede a salvo de la politización partidista, la cual ha sido y continúa siendo en algunos órdenes de gobierno, una forma evidente para fomentar el sistema de botín" (Uvalle, 2004; citado por Vega, 2016, p. 117).

Así la situación, se puede constatar que el rol que juegan los partidos políticos para allegar personal a la administración pública a través de la democracia electoral, es deficiente e insuficiente, por lo que se requiere transitar hacia otros esquemas o estrategias que mejoren esta situación, no solamente en el caso de México, sino también en el de la mayoría de países de América Latina y del mundo en general (Vega, 2016).

Pero a todo ello, ¿qué es la profesionalización del gobierno? La respuesta que emiten Aguilera y Bohórquez (2004; citados por Vega, 2016, p. 118) a esta interrogante es que "profesionalizar una administración pública es conseguir que los mejores hombres y mujeres de una sociedad encuentren los recursos y espacios necesarios para corregir, mejorar o transformar los bienes públicos existentes". Siguen exponiendo los citados autores que "profesionalizar es contar con los mejores cuadros para tomar las mejores decisiones públicas".

\section{La importancia del perfil profesional de los servidores públicos}

Para el tratamiento de este apartado, resulta pertinente mencionar las palabras de Gloria del Carmen Muñoz León, dentro del prólogo de la obra "La gestión de recursos humanos en las administraciones públicas estatales: aprendizajes y retos” (Instituto Nacional para el Federalismo y el Desarrollo Municipal [INAFED], 2006; citado por Vega, 2016, p. 118), al indicar que "el México actual nos exige empleados públicos con principios de neutralidad política, imparcialidad, objetividad e integridad moral, principios que configuran una 
administración profesional, dispuestos a desarrollar una óptima ejecución de las políticas públicas".

Un adecuado proceso de reclutamiento en la administración pública puede facilitar que lo anterior se alcance. En este sentido, un estudio de la OCDE (2011; citada por Vega, 2016, p. 118) indica que "una opción para establecer un proceso de reclutamiento flexible, orientado al desempeño [...] sería que las autoridades mexicanas consideraran la adopción de perfiles de puestos". Sigue señalando la OCDE que "éstos pueden ser un medio eficaz para que los ejecutivos y el personal piensen en sus funciones, lo que es importante en determinado puesto o conjunto de puestos, y de qué manera contribuye el empleo a lograr los objetivos de la institución".

Pero, ¿por qué es importante la creación de perfiles de puestos? Además de que son una herramienta de reclutamiento eficaz para atraer talentos al sector público, permiten la combinación de una declaración de lo que se espera de un empleo con una concepción de lo que quien ocupe el puesto debe aportar en aptitudes, experiencia, conductas y otros atributos necesarios para hacer bien el trabajo (OCDE, 2011; citada por Vega, 2016, p. 118).

Finalmente, ¿cómo se conceptualiza el término perfil de puesto? Se refiere al empleo; es decir, la finalidad del puesto, por qué existe y qué resultados se espera lograr para la organización pública de que se trate. El perfil de puesto también toma en cuenta a la persona, al hacer hincapié en las características que la institución busca en la persona que desempeñará determinado puesto. Es así que "cuando se implementan bien y se utilizan como parte de un conjunto integral de estrategias y procesos de gestión de recursos humanos, los perfiles de puestos son una herramienta de gran potencial (OCDE, 2011; citada por Vega, 2016 p. 118). 


\section{Reclutamiento político y acceso al poder público}

En palabras de Arce (2006; citado por Vega, 2016, p. 120), el reclutamiento político y el acceso al poder político, por lo general, se puede analizar por tres vías: a) acceder al poder por medio de los partidos políticos, lo que se ha denominado partidocracia; b) acceder al poder en dos órdenes: el federal y el estatal; y c) llegar al poder por medio de la administración pública.

El término partidocracia se ha utilizado para denotar un fenómeno de acaparamiento del poder político por un partido hegemónico o varios partidos en una coalición o en alianza, de manera que el proceso de toma de decisiones gubernamentales se realiza en el ámbito partidista, y por lo regular al margen de las esferas del gobierno (Arce, 2006; citado por Vega, 2016, p. 120).

Esta situación ha sido muy evidente en el caso de México y de muchas otras naciones del mundo. Es así que como bien lo señala Arce (2006), la partidocracia más que una forma de gobierno, es el dominio verdadero y propio de un partido político o de sus ambiciones de dominio; lo cual se ha criticado en el sentido de que esta forma de poder político se refiere a la permanencia de una élite de notables en puestos de elección popular, evitando, por medio de la reelección, la movilidad y estratificación social.

Sigue indicando el mencionado autor que "la partidocracia se identifica así por la preeminencia de los partidos en todos los ámbitos de la vida nacional: política, social y económica, que culmina en un complejo control de los partidos sobre la sociedad" (Arce, 2006; citado por Vega, 2016, p120).

Los políticos con una trayectoria en los gobiernos locales se han identificado como aquellos que tienen experiencia electoral-administrativa con influencia en el sistema político local (Arce, 2006; citado por Vega, 2016, p. 120). En términos del mismo Arce (2006), es evidente 
en una buena parte de los casos, que los políticos con trayectorias locales carecen de la suficiente preparación especializada en los asuntos nacionales e internacionales y se les señala como parciales frente a grupos de presión regionales; ya que en sus estados se les considera como una especie de caudillos que se benefician del amiguismo, de los lazos familiares y compadrazgos, y en la mayoría de los casos, sin una trayectoria política administrativa, ya sea en puestos de elección popular o en la administración pública local.

\section{Marco jurídico de los procesos políticos locales de San Luis Potosí Consideraciones preliminares}

En términos del artículo 26 fracción II de la CPSLP, son prerrogativas de los ciudadanos potosinos: I. Votar en las elecciones populares y consultas ciudadanas que lleven a cabo las autoridades competentes; y II. Poder ser votados para todos los cargos de elección popular y nombrados para ocupar cualquier empelo o comisión, teniendo las calidades que la CPSLP y las leyes aplicables establezcan [...]; Ejercer individual y libremente el derecho de asociarse y reunirse para tomar parte en forma pacífica en los asuntos políticos del Estado y los Municipios; y IV. Las demás que les confieran la CPSLP y las leyes que de ella emanen.

Siguiendo con el mismo artículo 26 de la CPSLP, éste señala que el derecho de solicitar el registro de candidatos ante la autoridad electoral corresponde a los partidos políticos, así como a los ciudadanos que soliciten su registro de manera independiente y cumplan con los requisitos, condiciones y términos que determine la legislación aplicable al caso.

Por lo que se refiere a los procesos electorales, el artículo 30 de la misma CPSLP señala que "corresponde a los ciudadanos, partidos políticos y al Consejo Estatal Electoral, la preparación, desarrollo y vigilancia de los procesos electorales, así como velar porque los 
mismos se lleven a cabo bajo los principios de, certeza, legalidad, imparcialidad, independencia, máxima publicidad, objetividad y equidad". En el mismo orden de ideas, "la calificación de las elecciones de Gobernador, diputados locales, y ayuntamientos, corresponde al Consejo Estatal Electoral y de Participación Ciudadana o, en su caso, al Instituto Nacional Electoral, de conformidad con la Constitución Federal y de acuerdo a las leyes federales y locales electorales" (artículo 31 de la CPSLP).

En cuanto al acceso de la clase política al poder, el artículo 36 de la CPSLP expone que "los partidos políticos son entidades de interés público que tienen como fin promover la participación de los ciudadanos en la vida democrática, y hacer posible el acceso de sus candidatos, mediante el sufragio universal, libre, secreto y directo de los electores, al ejercicio del poder público, de acuerdo con los programas, principios e ideas postulados por aquellos;

así como las reglas para garantizar la paridad entre los géneros, en candidaturas a diputados locales, y ayuntamientos".

En el mismo orden de ideas, el artículo 43 de la CPSLP puntualiza que "los partidos políticos con derecho a participar en las elecciones locales podrán postular un candidato para cada distrito uninominal y una lista de doce candidatos para ser electos por el principio de representación proporcional en la circunscripción estatal".

\section{Requisitos para ser diputado local}

Acorde con la CPSLP, para ser diputado local se requiere cumplir los requisitos que se muestran en el cuadro 1. 
Cuadro 1. Requisitos para ser diputado local en el Estado de San Luis Potosí.

I.- Ser ciudadano potosino en ejercicio de sus derechos;

II. Tener la calidad de potosino por nacimiento con residencia efectiva en el Estado no menor de seis meses inmediatos anteriores al día de la elección y, si se trata de potosino por vecindad, la residencia efectiva inmediata anterior al día de la elección deberá ser no menor de tres años, a partir de la adquisición de la calidad de vecino;

III. No tener una multa firme pendiente de pago, o que encontrándose sub júdice no esté garantizada en los términos de las disposiciones legales aplicables, que haya sido impuesta por responsabilidad con motivo de los cargos públicos que hubiere desempeñado en la administración federal, estatal o municipal; y no haber sido condenado por sentencia firme por la comisión de delitos dolosos que hayan ameritado pena de prisión; y

IV.- Tener como mínimo veintiún años de edad al día de la elección.

Fuente: Elaboración propia, a partir del artículo 46 de la CPSLP.

\section{Requisitos para ser presidente municipal}

En el cuadro 2 se muestran los requisitos que deben reunir las personas para ser presidente municipal en el Estado de San Luis Potosí.

\section{Cuadro 2. Requisitos para ser presidente municipal en el Estado de San Luis Potos}

I. Ser ciudadano potosino en ejercicio de sus derechos;

II. Ser originario del Municipio y con un año por lo menos de residencia efectiva en el mismo inmediata anterior a la fecha de la elección o designación, en su caso; o ser vecino del mismo, con residencia efectiva de dos años inmediata anterior al día de la elección o designación;

III. No tener una multa firme pendiente de pago, o que encontrándose sub júdice no esté garantizada en los términos de las disposiciones legales aplicables, que haya sido impuesta por responsabilidad con motivo de los cargos públicos que hubiere desempeñado en la administración federal, estatal o municipal; y no haber sido condenado por sentencia firme, por la comisión de delitos dolosos que hayan ameritado pena de prisión;

IV. No ser miembro de las fuerzas armadas o de policía que estén en servicio activo en el Estado, con cargo y atribuciones de mando en el municipio respectivo, a menos que separen de su cargo en el tiempo y forma que establece la ley de la materia, y

V. No ser ministro de culto religioso a menos que haya renunciado a su cargo en los términos que establece la ley de la materia.

Fuente: Elaboración propia, a partir del artículo 15 de la Ley Orgánica del Municipio Libre del Estado de San Luis Potosí. 


\section{Marco contextual del estudio}

\section{Datos generales sobre el desarrollo de los procesos electorales}

En las elecciones constitucionales de 2006 se registraron 102 candidatos a diputados locales con su respectivo suplente, participaron 9 partidos políticos: Partido Acción Nacional (PAN), Partido Revolucionario Institucional (PRI), Partido de la Revolución Democrática (PRD), Partido del Trabajo (PT), Partido Verde Ecologista de México (PVEM), Partido Conciencia Popular (PCP), Partido Convergencia (PC), Partido Nueva Alianza (PNA) y el Partido Alternativa Socialdemócrata y Campesina (PASC); así como 29 candidaturas comunes (CEE, 2007).

Según datos del CCE (2007), en el proceso electoral de 2006 participaron 3838 personas que se registraron en 230 planillas que encabezaron igual número de candidatos a presidentes municipales; 230 regidores de mayoría, 256 síndicos y 1318 regidores de representación proporcional, cada uno con su respectivo suplente. En el caso de la elección para presidentes municipales participaron 9 partidos políticos: PAN, PRI, PRD, PT, PVEM, PCP, PC, PNA, PASC y la Coalición por el Bien de Todos (CXBDT).

Por lo que se refiere al proceso electoral del 2009, se registraron 104 candidatos a diputado local con su respectivo suplente. Participaron 9 partidos políticos: PAN, PRI, PRD, PT, PVEM, PCP, PC, PNA y el PSD; hubo una Coalición que participó en los distritos V y X (Alianza en Acción) y 19 candidaturas comunes (CEEPAC, 2009).

En el proceso electoral del 2009 se registraron 258 planillas encabezadas por un candidato a presidente municipal, un regidor de mayoría con su suplente, participaron 264 candidatos a síndicos y 1996 a regidores de representación proporcional-cada uno con su respectivo sustituto-; hubo 68 candidaturas comunes. Para esta elección se inscribieron 5,294 personas (CEEPAC, 2009). 
Por lo que se refiere al proceso electoral del 2012, participaron 78 candidatos de mayoría relativa con su respectivo suplente, de los cuales 25 fueron mujeres (CEEPAC, 2013). Además, en términos del mismo CEEPAC, en las listas de representación proporcional hubo 96 candidatos, de los cuales 48 fueron mujeres.

Por lo que corresponde a la elección para presidentes municipales, en el proceso del 2012 participaron 235 candidatos con sus respectivas plantillas, de los cuales 33 fueron mujeres (CEEPAC, 2013).

\section{El entorno geográfico del Estado de San Luis Potosí}

El Estado de San Luis Potosí está situado en la zona centro-norte de la República Mexicana, entre $\operatorname{los} 21^{\circ} 09^{\prime} 30^{\prime \prime}$ y $24^{\circ} 33^{\prime} 09^{\prime \prime}$ latitud norte y $\operatorname{los} 98^{\circ} 19^{\prime} 52^{\prime \prime}$ y $102^{\circ} 17^{\prime} 51^{\prime \prime}$ de longitud al oeste de Greenwich. Sus límites geográficos son: a) Al Norte, con los estados de Coahuila, Nuevo León, Tamaulipas y Zacatecas. b) Al Sur, con Guanajuato, Hidalgo y Querétaro, c) Al Este, con Veracruz, d) Al Oeste, con Jalisco y Zacatecas (Vega, 2008).

Tiene una superficie de 62,304.74 kilómetros cuadrados (3.22 por ciento del territorio nacional) (Gobierno del Estado de SLP, Secretaría de Desarrollo Económico, 1999; citado por Vega, 2008). Su mayor longitud es de 430 kilómetros y su mayor anchura de 205. El Estado de San Luis Potosí está constituido por 58 municipios y está dividido geográficamente en cuatro zonas: Centro, Altiplano, Media y Huasteca. 


\section{Entorno político-electoral de San Luis Potosí}

El Estado de San Luis Potosí (SLP) está dividido en quince distritos electorales, los cuales se integran por cierto número de municipios (Tabla 1).

Tabla 1. Relación de distritos electorales del Estado de SLP.

\begin{tabular}{|c|c|c|}
\hline Distrito & Cabecera & Municipios que lo integran \\
\hline I & Matehuala & $\begin{array}{l}\text { Catorce, Cedral, Charcas, Matehuala, Vanegas, } \\
\text { Villa de Guadalupe y Villa de la Paz }\end{array}$ \\
\hline II & Cerritos & $\begin{array}{l}\text { Armadillo de los Infante, Cerritos, Guadalcázar, } \\
\text { San Nicolás Tolentino, Venado, Villa de Arista, } \\
\text { Villa Hidalgo y Villa Juárez }\end{array}$ \\
\hline III & Santa María del Río & $\begin{array}{l}\text { Cerro de San Pedro, Santa María del Río, Tierra } \\
\text { Nueva, Villa de Arriaga, Villa de Reyes y Zaragoza }\end{array}$ \\
\hline IV & Salinas & $\begin{array}{l}\text { Ahualulco, Mexquitic de Carmona, Moctezuma, } \\
\text { Salinas, Santo Domingo y Villa de Ramos }\end{array}$ \\
\hline $\begin{array}{l}\text { V, VI, VII y } \\
\text { VIII }\end{array}$ & San Luis Potosí & San Luis Potosí \\
\hline IX & $\begin{array}{l}\text { Soledad de Graciano } \\
\text { Sánchez }\end{array}$ & Soledad de Graciano Sánchez \\
\hline $\mathrm{X}$ & Rioverde & $\begin{array}{l}\text { Ciudad Fernández, Lagunillas, Rioverde y San Ciro } \\
\text { de Acosta }\end{array}$ \\
\hline XI & Cárdenas & $\begin{array}{l}\text { Alaquines, Cárdenas, Ciudad del Maíz, Rayón, } \\
\text { Santa Catarina, Tamasopo y El Naranjo }\end{array}$ \\
\hline XII & Ciudad Valles & Ciudad Valles \\
\hline XIII & Tamuín & $\begin{array}{l}\text { Coxcatlán, Ébano, San Antonio, San Vicente } \\
\text { Tancuayalab, Tampamolón Corona, Tamuín, } \\
\text { Tanlajás y Tanquián de Escobedo }\end{array}$ \\
\hline XIV & Tancanhuitz & $\begin{array}{l}\text { Aquismón, Tancanhuitz, Huehuetlán, Axtla de } \\
\text { Terrazas y Xilitla }\end{array}$ \\
\hline $\mathrm{XV}$ & Tamazunchale & $\begin{array}{l}\text { San Martín Chalchicuautla, Tamazunchale, } \\
\text { Tampacán y Matlapa }\end{array}$ \\
\hline
\end{tabular}

Fuente: Elaborado por el autor a partir de CEEPAC (2009). 


\section{Metodología}

Para la obtención de datos que permitieron construir los resultados que se presentan en este documento, se ejecutó una revisión y análisis de los datos biográficos de los diferentes actores que resultaron electos para los cargos de diputaciones de mayoría y de representación proporcional, así como de presidentes municipales, en los procesos políticos celebrados en el Estado de San Luis Potosí, durante los años 2006, 2009 y 2012.

El trabajo se apoyó en la información generada por el Consejo Estatal Electoral de San Luis Potosí (CEE), quien posteriormente cambió de nombre para quedar como Consejo Estatal Electoral y de Participación Ciudadana de San Luis Potosí (CEEPAC), a través de las obras editoriales: Los electos (2007, CEE) -derivada del proceso electoral 2006-, Los electos: proceso electoral 2009 (2009, CEEPAC), y Los electos: proceso electoral 2012 (2013, CEEPAC).

La revisión de datos quedó supeditada a la información emitida por el CEEPAC (anteriormente $\mathrm{CEE}$ ), tratándose de diputados de mayoría relativa y de representación proporcional; así como de los presidentes municipales electos en el 2006, 2009 у 2012.

Los datos tomados del CEEPAC para su revisión y análisis, consistieron en pequeñas biografías no estandarizadas de los actores políticos electos en los procesos electorales arriba señalados. En general, la información a la que se tuvo acceso para tratar de determinar los perfiles político y administrativo de los actores de referencia fue: a) lugar de nacimiento; b) fecha de nacimiento; c) nivel académico (en ciertos casos se pudo conocer el área profesional de estudio); d) experiencia académica, profesional o laboral; e) capacitación recibida (aunque no en todos los caso se tuvo la información). 
Para la construcción de los perfiles político y administrativo, se usó el esquema utilizado por Vega (2008), en el que como factores dentro del perfil administrativo se tienen: género, estado civil, año de nacimiento, lugar de nacimiento, profesión (o nivel de estudios y experiencia académica, laboral o profesional en la iniciativa privada (tratándose de experiencia en la administración pública, ésta forma parte del perfil político).

En cuanto al perfil político, se tomaron en cuenta factores como: actividad políticoestudiantil, actividad político-partidista, actividad político-sindical, afiliación partidista y su experiencia laboral en cualquier organización pública -federal, estatal o municipal-.

De los procesos electorales del 2006 se revisaron 85 biografías: 15 de diputados de mayoría relativa, 12 de diputados de representación proporcional y 58 de presidentes municipales. En el caso de las elecciones de 2009, la revisión fue la siguiente: 15 de diputados de mayoría relativa, 12 de diputados de representación proporcional y 58 de presidentes municipales. Por lo que hace al proceso político del 2012, se revisaron 85 biografías: 15 de diputados de mayoría relativa, 12 de diputados de representación proporcional y 58 de presidentes municipales. En total se revisaron 255 biografías de actores políticos electos en los tres comicios electorales.

Como el lector puede observar, se trató de un trabajo derivado totalmente de una revisión documental exclusivamente; ya que además de la bibliografía referida en el párrafo que antecede, se realizó búsqueda de información teórica en bases de datos a través de Internet; además de la consulta de diversas normas jurídicas, tanto locales como nacionales. 
Vol. 6, No. 11, 2016.

\section{Caso práctico: los procesos políticos 2006, 2009 y 2012 en San Luis Potosí}

\section{Resultados de la elección 2006}

Para el caso de las diputaciones locales de mayoría relativa, la Tabla 2 muestra los resultados obtenidos en el proceso electoral de que se trata.

Tabla 2. Resultados electorales para diputados locales de mayoría relativa de SLP en 2006.

\begin{tabular}{|c|c|c|}
\hline $\begin{array}{r}\text { Distrito } \\
\text { electoral }\end{array}$ & $\begin{array}{l}\text { Partido (s) político postulante } \\
\text { vencedor }\end{array}$ & Número de votos obtenidos \\
\hline $\mathrm{I}$ & PAN & 25,253 \\
\hline II & PAN, PVEM y PNA & $\begin{array}{l}\text { PAN }=18,734 ; \text { PVEM }=1,072 ; \text { PNA }=621 \mathrm{y} \\
\text { PAN-PVEM-PNA }=1,299\end{array}$ \\
\hline III & PAN y PNA & $\begin{array}{l}\text { PAN }=21,701 ; \text { PNA }=1,158 \text { y PAN-PNA }= \\
2,049\end{array}$ \\
\hline IV & PRD y PT & $\mathrm{PRD}=5,199 ; \mathrm{PT}=11,172$ y $\mathrm{PRD}-\mathrm{PT}=1,953$ \\
\hline $\mathrm{V}$ & PAN & 37,669 \\
\hline VI & PAN & 27,542 \\
\hline VII & PAN & 41,341 \\
\hline VIII & PAN & 40,523 \\
\hline IX & PAN & 29,455 \\
\hline $\mathrm{X}$ & PAN y PNA & $\begin{array}{l}\text { PAN }=24,927 ; \text { PNA }=2,396 \text { y PAN-PNA }= \\
1,913\end{array}$ \\
\hline $\mathrm{XI}$ & PAN, PVEM y PNA & $\begin{array}{l}\text { PAN }=18,113 ; \text { PVEM }=404 ; \text { PNA }=847 \mathrm{y} \\
\text { PAN-PVEM-PNA }=538\end{array}$ \\
\hline XII & PAN & 26,659 \\
\hline XIII & PAN y PVEM & $\begin{array}{l}\text { PAN }=21,878 ; \text { PVEM }=632 \text { y PAN-PVEM = } \\
563\end{array}$ \\
\hline XIV & PAN y PVEM & $\begin{array}{l}\text { PAN }=24,951 ; \text { PVEM }=688 \text { y PAN-PVEM = } \\
749\end{array}$ \\
\hline $\mathrm{XV}$ & $\begin{array}{lll}\text { PRI y Partido } & \text { Alternativa } \\
\begin{array}{l}\text { Socialdemócrata y } \\
\text { (PASC) }\end{array} & \text { Campesina } \\
\end{array}$ & $\begin{array}{l}\text { PRI }=16,121 ; \text { PASC }=1,410 \text { y PRI-PASC }= \\
658\end{array}$ \\
\hline
\end{tabular}

Fuente: Elaborada por el autor a partir de CEE (2007). 
En cuanto a los diputados de representación proporcional, la legislatura correspondiente quedó integrada de la siguiente manera: 2 diputados por el PAN; 4 por el PRI, 3 por el PRD; uno por el PT; uno por el PVEM; y uno por el PCP.

Los resultados electorales para elegir a los 58 presidentes municipales de la entidad en las elecciones de 2006, se muestran en la Tabla 3.

Tabla 3. Resultados electorales para presidentes municipales del Estado de SLP para el periodo 2007-2009.

\begin{tabular}{|c|c|c|}
\hline Municipio & $\begin{array}{c}\text { Partido (s) } \\
\text { político (s) } \\
\text { postulante (s) } \\
\text { vencedor }(\mathrm{es}) \\
\end{array}$ & Número de votos obtenidos \\
\hline Ahualulco & PAN & 2,269 \\
\hline Alaquines & PAN, PCP y PNA & $\begin{array}{l}\text { PAN }=497 ; \text { PCP }=971 ; \text { PNA }=122 \text { y PAN-PCP- } \\
\text { PNA }=101\end{array}$ \\
\hline Aquismón & PAN & 8,256 \\
\hline $\begin{array}{l}\text { Armadillo de los } \\
\text { Infante }\end{array}$ & PRI y PASC & $\mathrm{PRI}=712 ; \mathrm{PASC}=21$ y PRI-PASC $=712$ \\
\hline Axtla de Terrazas & PAN & 5,512 \\
\hline Cárdenas & PRI & 2,925 \\
\hline Catorce & PAN & 2,230 \\
\hline Cedral & PAN & 2,317 \\
\hline Cerritos & PRI & 4,148 \\
\hline Cerro de San Pedro & PCP & 909 \\
\hline Charcas & PRI y PASC & PRI $=2,798 ;$ PASC $=139$ y PRI-PASC $=143$ \\
\hline Ciudad del Maíz & PRI & 6,335 \\
\hline Ciudad Fernández & PRI & 5,191 \\
\hline Ciudad Valles & PAN & 22,530 \\
\hline Coxcatlán & PRI & 2,554 \\
\hline Ébano & PAN & 5,963 \\
\hline El Naranjo & PAN y PNA & $\mathrm{PAN}=3,529 ; \mathrm{PNA}=160$ y PAN-PNA $=286$ \\
\hline Guadalcázar & PRI & 5,205 \\
\hline Huehuetlán & PAN & 3,190 \\
\hline Lagunillas & PAN & 1,124 \\
\hline Matehuala & PAN & 20,998 \\
\hline Matlapa & PAN & 5,560 \\
\hline Mexquitic de Carmona & PRI & 7,504 \\
\hline Moctezuma & PCP & 1,925 \\
\hline Rayón & PRI & 2,697 \\
\hline Rioverde & PAN y PNA & PAN $=16,838 ;$ PNA $=1,051$ y PAN-PNA $=2,672$ \\
\hline
\end{tabular}


Vol. 6, No. 11, 2016

\begin{tabular}{|c|c|c|}
\hline Salinas & PAN y PNA & PAN $=3,880 ;$ PNA $=270$ y PAN-PNA $=377$ \\
\hline San Antonio & PAN & 1,988 \\
\hline San Ciro de Acosta & PRI & 2,385 \\
\hline San Luis Potosí & PAN y PNA & PAN $=152,398 ;$ PNA $=7,360$ y PAN-PNA $=2,104$ \\
\hline $\begin{array}{ll}\text { San } & \text { Martín } \\
\text { Chalchicuautla } & \end{array}$ & $\begin{array}{l}\text { Coalición por el } \\
\text { Bien de Todos } \\
\text { (PRD, PT y } \\
\text { Partido } \\
\text { Convergencia } \\
\text { [CXBT] })\end{array}$ & 3,250 \\
\hline San Nicolás Tolentino & CXBT & 966 \\
\hline $\begin{array}{ll}\text { San } & \text { Vicente } \\
\text { Tancuayalab } & \end{array}$ & PCP & 2,468 \\
\hline Santa Catarina & CXBT & 1,156 \\
\hline Santa María del Río & PRI, PCP y PASC & $\begin{array}{l}\mathrm{PRI}=5,151 ; \mathrm{PCP}=84 ; \text { PASC }=103 \text { y PRI-PCP- } \\
\text { PASC }=505\end{array}$ \\
\hline Santo Domingo & PAN & 2,124 \\
\hline $\begin{array}{l}\text { Soledad de Graciano } \\
\text { Sánchez }\end{array}$ & PAN y PNA & $\mathrm{PAN}=23,170 ; \mathrm{PNA}=1,845$ y $\mathrm{PAN}-\mathrm{PNA}=2,307$ \\
\hline Tamasopo & PAN y PNA & $\mathrm{PAN}=5,157 ;$ PNA $=215$ y PAN-PNA $=478$ \\
\hline Tamazunchale & PRI & 13,067 \\
\hline Tampacán & PRI y PASC & $\mathrm{PRI}=2,238 ; \mathrm{PASC}=1,084$ y PRI-PASC $=106$ \\
\hline Tampamolón Corona & PAN & 2,591 \\
\hline Tamuín & PAN & 4,919 \\
\hline Tancanhuitz & PRI & 3,095 \\
\hline Tanlajás & PAN & 2,443 \\
\hline Tanquián de Escobedo & PAN & 3,242 \\
\hline Tierra Nueva & PRI y PASC & PRI $=1,604 ;$ PASC $=88$ y PRI-PASC $=280$ \\
\hline Vanegas & PRI y PASC & PRI $=1,469 ;$ PASC $=21$ y PRI-PASC $=292$ \\
\hline Venado & PRI & 2,213 \\
\hline Villa de Arista & PRI y PASC & PRI $=1,942 ;$ PASC $=57$ y PRI-PASC $=298$ \\
\hline Villa de Arriaga & CXBT & 2,089 \\
\hline Villa de Guadalupe & PAN & 1,683 \\
\hline Villa de la Paz & PRI y PASC & $\mathrm{PRI}=1,200 ; \mathrm{PASC}=31$ y PRI-PASC $=62$ \\
\hline Villa de Ramos & PRI & 5,123 \\
\hline Villa de Reyes & PAN y PNA & PAN =7,218; PNA =413 y PAN-PNA =914 \\
\hline Villa Hidalgo & PAN y PNA & PAN $=2,838 ;$ PNA $=175$ y PAN-PNA =339 \\
\hline Villa Juárez & PRI y PASC & PRI $=2,021 ;$ PASC $=36$ y PRI-PASC $=303$ \\
\hline Xilitla & PRI & 8,499 \\
\hline Zaragoza & PAN y PNA & $\mathrm{PAN}=2,585 ; \mathrm{PNA}=166$ y PAN-PNA $=275$ \\
\hline
\end{tabular}

Fuente: Elaborada por el autor a partir de CEE (2007). 


\section{Resultados de la elección 2009}

Para el caso de las diputaciones locales de mayoría relativa, la Tabla 4 muestra los resultados obtenidos en el proceso electoral de que se trata.

Tabla 4. Resultados electorales para diputados locales de mayoría relativa de SLP en 2009.

\begin{tabular}{|l|l|l|}
\hline $\begin{array}{c}\text { Distrito } \\
\text { electoral }\end{array}$ & \multicolumn{1}{|c|}{$\begin{array}{c}\text { Partido (s) político (s) } \\
\text { postulante (s) vencedor }\end{array}$} & \multicolumn{1}{c|}{ Número de votos obtenidos } \\
\hline I & PRI & 25,160 \\
\hline II & PRI & 21,286 \\
\hline III & PAN y PNA & PAN $=19,759 ;$ PNA $=2,645$ y PAN-PNA $=1,113$ \\
\hline IV & PAN y PNA & PAN $=19,339 ;$ PNA $=1,645$ y PAN-PNA $=2,361$ \\
\hline V & PRI & 22,062 \\
\hline VI & PAN y PNA & PAN $=19,853 ;$ PNA $=3,070$ y PAN-PNA $=1,192$ \\
\hline VII & PAN y PNA & PAN $=30,699 ;$ PNA $=3047$ y PAN-PNA $=2,164$ \\
\hline VIII & PRI y PVEM & PRI $=20,658 ;$ PVEM $=8,286$ y PRI-PVEM $=786$ \\
\hline IX & PAN y PNA & PAN $=14,806 ;$ PNA $=2,485$ y PAN-PNA $=1,115$ \\
\hline X & Alianza en Acción $($ PAN y & 29,304 \\
\hline XI & PNA) & 22,297 \\
\hline XII & PRI & PAN $=17,492 ;$ PNA $=1,276$ y PAN-PNA $=1,269$ \\
\hline XIII & PRI y PSD & PRI $=24,362 ;$ PSD $=1,133$ y PRI-PSD $=1,633$ \\
\hline XIV & PAN y PNA & PAN $=22,921 ;$ PNA $=950$ y PAN-PNA $=1,931$ \\
\hline XV & PAN y PNA & PAN $=21,604 ;$ PNA $=5,241$ y PAN-PNA $=1,602$ \\
\hline
\end{tabular}

Fuente: Elaborada por el autor a partir de CEEPAC (2009).

En cuanto a los diputados de representación proporcional para el periodo 2009.2012, la legislatura correspondiente quedó integrada de la siguiente manera: 3 diputados por el PAN; 3 por el PRI, uno por el PRD; uno por el PT; uno por el PVEM; uno por el PCP y 2 por el PNA (CEEPAC, 2009).

Los resultados electorales para elegir a los 58 presidentes municipales de la entidad en las elecciones de 2009, se muestran en la Tabla 5. 
Tabla 5. Resultados electorales para presidentes municipales del Estado de SLP para el periodo 2009-2012.

\begin{tabular}{|c|c|c|}
\hline Municipio & $\begin{array}{c}\text { Partido (s) } \\
\text { político (s) } \\
\text { postulante (s) } \\
\text { vencedor }(\mathrm{es}) \\
\end{array}$ & Número de votos obtenidos \\
\hline Ahualulco & PRI & 2,370 \\
\hline Alaquines & PAN & 1,930 \\
\hline Aquismón & PAN y PNA & PAN $=6,516 ;$ PNA $=111$ y PAN-PNA $=722$ \\
\hline $\begin{array}{l}\text { Armadillo de los } \\
\text { Infante }\end{array}$ & PRI & 1,075 \\
\hline Axtla de Terrazas & PRI & 7,338 \\
\hline Cárdenas & PAN, PCP y PNA & $\begin{array}{l}\text { PAN }=1,624 ; \text { PCP }=1,466 ; \text { PNA }=495 \text { y PAN-PCP- } \\
\text { PNA }=423\end{array}$ \\
\hline Catorce & PRI & 1,712 \\
\hline Cedral & PRI & 4,452 \\
\hline Cerritos & PT y PC & $\mathrm{PT}=2,768 ; \mathrm{PC}=591$ y $\mathrm{PT}-\mathrm{PC}=293$ \\
\hline Cerro de San Pedro & PAN y PNA & PAN $=824 ;$ PNA $=69$ y PAN-PNA $=132$ \\
\hline Charcas & PAN y PNA & $\mathrm{PAN}=3,347 ; \mathrm{PNA}=169$ y PAN-PNA $=502$ \\
\hline Ciudad del Maíz & PRI & 7,138 \\
\hline Ciudad Fernández & PAN y PNA & PAN $=8,208 ;$ PNA =767 y PAN-PNA =388 \\
\hline Ciudad Valles & PAN, PCP y PNA & $\begin{array}{l}\text { PAN }=19,012 ; \text { PCP }=768 ; \text { PNA }=1,102 \text { y PAN-PCP- } \\
\text { PNA }=2,553\end{array}$ \\
\hline Coxcatlán & PAN y PNA & PAN =2,160; PNA =61 y PAN-PNA =263 \\
\hline Ébano & PRI y PSD & PRI =6,046; PSD =181 y PRI-PSD =694 \\
\hline El Naranjo & PRI y PVEM & PRI =3,337; PVEM =284 y PRI-PVEM =162 \\
\hline Guadalcázar & PRI & 5,424 \\
\hline Huehuetlán & PRI & 3,645 \\
\hline Lagunillas & PRI & 1,131 \\
\hline Matehuala & PAN y PNA & PAN $=11,942 ;$ PNA $=1,065$ y PAN-PNA $=1,024$ \\
\hline Matlapa & PAN & 4,074 \\
\hline $\begin{array}{l}\text { Mexquitic de } \\
\text { Carmona }\end{array}$ & PAN y PNA & $\mathrm{PAN}=6,279 ; \mathrm{PNA}=444$ y PAN-PNA =1,197 \\
\hline Moctezuma & PAN y PNA & PAN =3,016; PNA =171 y PAN-PNA =533 \\
\hline Rayón & PRD & 2,981 \\
\hline Rioverde & PAN y PNA & PAN $=17,450 ;$ PNA $=1,775$ y PAN-PNA $=1,040$ \\
\hline Salinas & PAN y PNA & PAN =2,696; PNA =258 y PAN-PNA =227 \\
\hline San Antonio & PAN & 2,150 \\
\hline San Ciro de Acosta & PAN & 2,316 \\
\hline San Luis Potosí & PRI y PVEM & PRI =93,627; PVEM =18,847 y PRI-PVEM =3,409 \\
\hline
\end{tabular}


Vol. 6, No. 11, 2016.

\begin{tabular}{|c|c|c|}
\hline $\begin{array}{l}\text { San Martín } \\
\text { Chalchicuautla }\end{array}$ & PAN y PNA & $\mathrm{PAN}=5,069 ; \mathrm{PNA}=149$ y PAN-PNA $=509$ \\
\hline $\begin{array}{l}\text { San Nicolás } \\
\text { Tolentino }\end{array}$ & PCP & 912 \\
\hline $\begin{array}{l}\text { San Vicente } \\
\text { Tancuayalab }\end{array}$ & PT & 1,620 \\
\hline Santa Catarina & PAN, PT y PNA & $\begin{array}{l}\text { PAN }=365 ; \text { PT }=1,110 ; \text { PNA }=107 \text { y PAN-PT-PNA } \\
=295\end{array}$ \\
\hline $\begin{array}{l}\text { Santa } \text { María del } \\
\text { Río }\end{array}$ & PAN & 9,048 \\
\hline Santo Domingo & PRD y PT & $\mathrm{PRD}=199 ; \mathrm{PT}=1881$ y PRD-PT $=540$ \\
\hline $\begin{array}{l}\text { Soledad de } \\
\text { Graciano Sánchez }\end{array}$ & $\begin{array}{l}\text { PRD, PT, PCP y } \\
\text { PSD }\end{array}$ & $\begin{array}{l}\text { PRD }=15,440 ; \text { PT }=2,775 ; \text { PCP }=3,692 ; P S D=1,391 \text { y } \\
\text { PRD-PT-PCP-PSD }=2,543\end{array}$ \\
\hline Tamasopo & PRI & 4,285 \\
\hline Tamazunchale & PAN y PNA & $\mathrm{PAN}=10,843 ; \mathrm{PNA}=5,942$ y $\mathrm{PAN}-\mathrm{PNA}=1,534$ \\
\hline Tampacán & PRI y PNA & PRI =3,182; PNA =206 y PRI-PNA =82 \\
\hline $\begin{array}{l}\text { Tampamolón } \\
\text { Corona }\end{array}$ & PRI y PVEM & PRI =2,3304; PVEM =126 y PRI-PVEM =392 \\
\hline Tamuín & PNA & 4,893 \\
\hline Tancanhuitz & PAN & 3,423 \\
\hline Tanlajás & PRD & 3,130 \\
\hline $\begin{array}{l}\text { Tanquián de } \\
\text { Escobedo }\end{array}$ & PRI & 3,607 \\
\hline Tierra Nueva & PRI & 2,092 \\
\hline Vanegas & PAN y PNA & $\mathrm{PAN}=1,352 ; \mathrm{PNA}=79$ y PAN-PNA $=130$ \\
\hline Venado & PAN & 2,234 \\
\hline Villa de Arista & PAN & 2,886 \\
\hline Villa de Arriaga & PRI & 2,716 \\
\hline Villa de Guadalupe & PRD & 1,291 \\
\hline Villa de la Paz & PCP & 1,045 \\
\hline Villa de Ramos & PRI & 4,252 \\
\hline Villa de Reyes & PCP & 6,244 \\
\hline Villa Hidalgo & PRI & 4,305 \\
\hline Villa Juárez & PAN y PNA & $\mathrm{PAN}=2,410 ; \mathrm{PNA}=58$ y $\mathrm{PAN}-\mathrm{PNA}=234$ \\
\hline Xilitla & PRI & 7,309 \\
\hline Zaragoza & PVEM y PCP & $\mathrm{PVEM}=3,170 ; \mathrm{PCP}=474$ y $\mathrm{PVEM}-\mathrm{PCP}=283$ \\
\hline
\end{tabular}

Fuente: Elaborada por el autor a partir de CEEPAC (2009). 


\section{Resultados de la elección 2012}

Para el caso de las diputaciones locales de mayoría relativa, la Tabla 6 muestra los resultados obtenidos en el proceso electoral de que se trata.

Tabla 6. Resultados electorales para diputados locales de mayoría relativa de SLP en 2012.

\begin{tabular}{|l|l|l|}
\hline $\begin{array}{c}\text { Distrito } \\
\text { electoral }\end{array}$ & \multicolumn{1}{|c|}{$\begin{array}{c}\text { Partido (s) político (s) } \\
\text { postulante (s) vencedor } \\
\text { (es) }\end{array}$} & \multicolumn{1}{c|}{ Número de votos obtenidos } \\
\hline I & PRI y PVEM & PRI $=28,201 ;$ PVEM $=4,054$ \\
\hline II & PRI & 19,753 \\
\hline III & PRI y PVEM & PRI $=18,456 ;$ PVEM $=3,365$ \\
\hline IV & PRI y PVEM & PRI $=19,942 ;$ PVEM $=3,270$ \\
\hline V & PRI y PVEM & PRI $=19,295 ;$ PVEM $=4,935$ \\
\hline VI & PAN y PNA & PAN-PNA $=33,126$ \\
\hline VII & PAN y PNA & PAN $=41,405 ;$ PNA $=2,328$ y PAN-PNA $=3,038$ \\
\hline VIII & PAN y PNA & PAN-PNA $=23,763$ \\
\hline IX & PRD y PT & PRD $=21,253 ;$ PT $=1,931$ y PRD-PT $=5,579$ \\
\hline X & PRI y PVEM & PRI $=24,119 ;$ PVEM $=1,549$ y PRI-PVEM $=3,505$ \\
\hline XI & PRI y PVEM & PRI $=19,033 ;$ PVEM $=1,687$ y PRI-PVEM $=4,322$ \\
\hline XII & PRI y PVEM & PRI-PVEM $=32,515$ \\
\hline XIII & PRI y PVEM & PRI $=22,021 ;$ PVEM $=2,142$ y PRI-PVEM $=3,445$ \\
\hline XIV & PAN y PNA & PAN $=22,273 ;$ PNA $=1,760$ y PAN-PNA $=2,385$ \\
\hline XV & PRI y PVEM & PRI-PVEM $=28,431$ \\
\hline
\end{tabular}

Fuente: Elaborada por el autor a partir de CEEPAC (2013).

En cuanto a los diputados de representación proporcional para el periodo 2012-2015, la legislatura correspondiente quedó integrada de la siguiente manera: 2 diputados por el PAN; 2 por el PRI, uno por el PRD; uno por el PT; 2 por el PVEM; uno por el PCP, uno por el Partido Movimiento Ciudadano (PMC) y 2 por el PNA (CEEPAC, 2013).

Los resultados electorales para elegir a los 58 presidentes municipales de la entidad en las elecciones de 2012, se muestran en la Tabla 7. 
Tabla 7. Resultados electorales para presidentes municipales del Estado de SLP para el periodo 2012-2015.

\begin{tabular}{|c|c|c|}
\hline Municipio & $\begin{array}{c}\text { Partido (s) } \\
\text { político (s) } \\
\text { postulante (s) } \\
\text { vencedor (es) }\end{array}$ & Número de votos obtenidos \\
\hline Ahualulco & PAN y PNA & $\mathrm{PAN}=2,032 ; \mathrm{PNA}=65 ;$ PAN-PNA $=587$ \\
\hline Alaquines & PRI y PVEM & PRI-PVEM $=1,759$ \\
\hline Aquismón & PAN y PNA & PAN $=7,903 ;$ PNA $=174$ y PAN-PNA $=1,166$ \\
\hline $\begin{array}{l}\text { Armadillo de los } \\
\text { Infante }\end{array}$ & PRI y PVEM & PRI =746; PVEM =38 y PRI-PVEM =333 \\
\hline Axtla de Terrazas & PRI y PVEM & PRI-PVEM =3560 \\
\hline Cárdenas & PRI y PVEM & PRI-PVEM =4,126 \\
\hline Catorce & PRI y PVEM & PRI =1,494; PVEM =127 y PRI-PVEM =705 \\
\hline Cedral & PRI y PVEM & PRI-PVEM $=4,978$ \\
\hline Cerritos & PAN y PNA & PAN $=3,930 ;$ PNA $=153$ y PAN-PNA $=572$ \\
\hline $\begin{array}{lll}\text { Cerro } & \text { de } & \text { San } \\
\text { Pedro } & & \\
\end{array}$ & PRI & 1,269 \\
\hline Charcas & PRI y PVEM & PRI-PVEM =4,380 \\
\hline Ciudad del Maíz & PRI y PVEM & PRI-PVEM =7,593 \\
\hline $\begin{array}{l}\text { Ciudad } \\
\text { Fernández }\end{array}$ & PT & 8,327 \\
\hline Ciudad Valles & PRI y PVEM & PRI =22,165; PVEM =2,067; PRI-PVEM =5,375 \\
\hline Coxcatlán & PRI y PVEM & PRI =1,959; PVEM =158; PRI-PVEM =686 \\
\hline Ébano & PRI y PVEM & PRI-PVEM $=6,163$ \\
\hline El Naranjo & PRI y PVEM & PRI =2,340; PVEM =137 y PRI-PVEM =622 \\
\hline Guadalcázar & PRI y PVEM & PRI-PVEM =6,267 \\
\hline Huehuetlán & PRI y PVEM & PRI =558; PVEM =1,490 y PRI-PVEM =644 \\
\hline Lagunillas & PRI y PVEM & PRI-PVEM =1,300 \\
\hline Matehuala & PRI y PVEM & $\mathrm{PRI}=11,829 ; \mathrm{PVEM}=557$ y PRI-PVEM $=3,534$ \\
\hline Matlapa & PRI y PVEM & PRI =5,542; PVEM =372 y PRI-PVEM =1,598 \\
\hline $\begin{array}{l}\text { Mexquitic de } \\
\text { Carmona }\end{array}$ & PRI y PVEM & PRI =7,174; PVEM =438 y PRI-PVEM =3,529 \\
\hline Moctezuma & PCP & 2,788 \\
\hline Rayón & PRI y PVEM & PRI-PVEM =2,593 \\
\hline Rioverde & PRI y PVEM & PRI =16,678; PVEM =893 y PRI-PVEM =2,938 \\
\hline Salinas & PRD, PT y PMC & $\begin{array}{l}\mathrm{PRD}=1,051 ; \mathrm{PT}=2,204 ; \mathrm{PMC}=260 \mathrm{y} \text { PRD-PT-PMC } \\
=2,130\end{array}$ \\
\hline San Antonio & PRI y PVEM & PRI-PVEM =2,368 \\
\hline
\end{tabular}


Vol. 6, No. 11, 2016.

\begin{tabular}{|c|c|c|}
\hline $\begin{array}{l}\text { San Ciro de } \\
\text { Acosta }\end{array}$ & PRI y PVEM & PRI =1,833; PVEM =71 y PRI-PVEM =676 \\
\hline San Luis Potosí & PRI y PVEM & PRI-PVEM $=137,257$ \\
\hline $\begin{array}{l}\text { San Martín } \\
\text { Chalchicuautla }\end{array}$ & PAN y PNA & PAN $=3,607 ;$ PNA $=184$ y PAN-PNA $=370$ \\
\hline $\begin{array}{l}\text { San Nicolás } \\
\text { Tolentino }\end{array}$ & PAN & 846 \\
\hline $\begin{array}{l}\text { San Vicente } \\
\text { Tancuayalab }\end{array}$ & PRI, PVEM y PCP & $\begin{array}{l}\text { PRI }=3,184 ; \text { PVEM }=127 ; \text { PCP }=105 \text { y PRI-PVEM- } \\
\text { PCP }=859\end{array}$ \\
\hline Santa Catarina & PRI y PVEM & PRI-PVEM =2,271 \\
\hline $\begin{array}{l}\text { Santa María del } \\
\text { Río }\end{array}$ & PRI y PVEM & PRI =5,162; PVEM =311 y PRI-PVEM =2,235 \\
\hline Santo Domingo & PCP & $\mathrm{PRD}=2,502$ \\
\hline $\begin{array}{l}\text { Soledad } \mathrm{de} \\
\text { Graciano Sánchez }\end{array}$ & PRD, PT y PMC & $\begin{array}{l}\text { PRD }=31,861 ; \text { PT }=2,326 ; \text { PMC }=2,311 ; \text { PRD-PT- } \\
\text { PMC }=18,006\end{array}$ \\
\hline Tamasopo & PRI y PVEM & PRI =4,114; PVEM =529 y PRI-PVEM =1,228 \\
\hline Tamazunchale & PNA & 14,914 \\
\hline Tampacán & PRD y PCP & $\mathrm{PRD}=2,752 ; \mathrm{PCP}=338$ y $\mathrm{PRD}-\mathrm{PCP}=332$ \\
\hline $\begin{array}{l}\text { Tampamolón } \\
\text { Corona }\end{array}$ & PAN y PNA & PAN $=2,037 ;$ PNA $=154$ y PAN-PNA $=513$ \\
\hline Tamuín & PRI y PVEM & PRI-PVEM $=5,202$ \\
\hline Tancanhuitz & PRI-PCP & $\mathrm{PRI}=3,571 ; \mathrm{PCP}=101$ y PRI-P \\
\hline Tanlajás & PT & 3,280 \\
\hline $\begin{array}{l}\text { Tanquián de } \\
\text { Escobedo }\end{array}$ & PAN y PNA & PAN $=2,410 ;$ PNA $=198$ y PAN-PNA $=790$ \\
\hline Tierra Nueva & PCP & 1,839 \\
\hline Vanegas & PRI y PVEM & PRI =434; PVEM =1,075 y PRI-PVEM =706 \\
\hline Venado & PAN y PNA & PAN $=2,447 ;$ PNA $=111$ y PAN-PNA $=633$ \\
\hline Villa de Arista & PRI y PVEM & PRI =3,278; PVEM =991 y PRI-PVEM =1,215 \\
\hline Villa de Arriaga & $\begin{array}{l}\text { PAN, PRD, PT, } \\
\text { PVEM, PCP, PMC } \\
\text { y PNA }\end{array}$ & $\begin{array}{l}\text { PAN }=1264 ; \text { PRD }=217 ; \text { PT }=69 ; \text { PVEM }=1,362 ; \text { PCP } \\
=59 ; \text { PMC }=128 ; \text { PNA }=570 \text { y PAN-PRD-PT-PVEM- } \\
\text { PCP-PMC-PNA }=1,013\end{array}$ \\
\hline $\begin{array}{ll}\text { Villa de } \\
\text { Guadalupe }\end{array}$ & PRI y PVEM & PRI-PVEM =1,786 \\
\hline Villa de la Paz & PAN y PNA & $\mathrm{PAN}=5932 ; \mathrm{PNA}=40 \mathrm{y}$ PAN-PNA $=177$ \\
\hline Villa de Ramos & PRD, PT y PMC & $\begin{array}{l}\text { PRD }=1,102 ; \mathrm{PT}=2,711 ; \mathrm{PMC}=203 \text { y PRD-PT-PMC } \\
=1,901\end{array}$ \\
\hline Villa de Reyes & $\mathrm{PCP}$ & 7,069 \\
\hline Villa Hidalgo & PRI & 3,564 \\
\hline Villa Juárez & PAN y PNA & PAN $=1,740 ;$ PNA $=122$ y PAN-PNA $=499$ \\
\hline Xilitla & PRI Y PVEM & PRI =8,071; PVEM =472 y PRI-PVEM =3,080 \\
\hline Zaragoza & PT y PMC & $\mathrm{PT}=1,140 ; \mathrm{PMC}=953$ y $\mathrm{PT}-\mathrm{PMC}=1,081$ \\
\hline
\end{tabular}

Fuente: Elaborada por el autor a partir de CEEPAC (2013). 


\section{Revisión y análisis de los datos biográficos de los sujetos de estudio Perfil profesional de los actores electos en el proceso político 2006}

Por lo que se refiere a los procesos electorales del 2006, se encontraron los siguientes resultados del análisis de los perfiles político y administrativo de los servidores públicos electos:

- Diputados locales de mayoría relativa

De los 15 diputados electos, es de llamar la atención que un alto porcentaje de los mismos son del sexo masculino (86.66\%) y solamente dos del sexo femenino (13.33\%). El 80\% de los diputados cuentan con el nivel educativo de licenciatura / ingeniería. Un diputado tiene estudios de preparatoria y dos diputados apenas cuentan con educación secundaria.

Las áreas del conocimiento que destacan en la preparación académica de los sujetos analizados son agronomía y derecho. Solamente un diputado cuenta con una preparación relacionada con la administración pública.

Por lo que se refiere a los partidos políticos postulantes de los diputados, tenemos que el PAN cuenta con el $86.66 \%$ de los curules, aunque para ello tuvo que realizar alianzas con los partidos PVEM y PNA. El PRI cuenta con una diputación (en alianza con el PASC) y el PRD con otra (en coalición con el PT). En el caso de los 15 diputados electos, todos contaban con experiencia laboral en el sector público y en la mayor parte de los casos, se observa una relación directa y constante con el partido que los postuló.

\section{- Diputados locales de representación proporcional}


En el caso de los 12 diputados electos, la proporción en cuanto a género es más o menos equilibrada, ya que son 5 personas del sexo femenino por 7 del masculino. En lo que se refiere al nivel académico, la proporción de estudios está entre preparatoria / bachillerato / carrera técnica y licenciatura ( 5 y 6 respectivamente). Uno de los diputados cuenta con estudios de postgrado (maestría).

El área de conocimiento que predomina entre los diputados electos, es la profesión de derecho. Los diputados electos pertenecen a los siguientes partidos políticos: dos al PAN, cuatro al PRI, tres al PRD, uno al PT, uno al PVEM y uno al PCP.

\section{- Presidentes municipales}

En el proceso electoral que se analiza, se eligieron 58 presidentes municipales. Nuevamente llama la atención que de los actores políticos electos, el 93.10\% son del sexo masculino (54), contra un $6.89 \%$ que pertenecen al sexo femenino (4). La formación académica de los servidores electos se muestra en la Tabla 8.

Tabla 8. Nivel académico de los presidentes municipales electos en el proceso electoral del 2006 en el Estado de SLP.

\begin{tabular}{|l|c|c|}
\hline \multicolumn{1}{|c|}{ Nivel académico concluido } & Cantidad & $\begin{array}{c}\text { Porcentaje del total de presidentes } \\
\text { municipales electos }\end{array}$ \\
\hline Licenciatura / ingeniería & 28 & $48.27 \%$ \\
\hline $\begin{array}{l}\text { Bachillerato / preparatoria / carrera } \\
\text { técnica }\end{array}$ & 14 & $24.13 \%$ \\
\hline Secundaria & 8 & $13.79 \%$ \\
\hline Primaria & 7 & $12.06 \%$ \\
\hline Primaria inconclusa (4 $4^{\circ}$ grado) & 1 & $1.72 \%$ \\
\hline
\end{tabular}

Fuente: Elaborada por el autor a partir de CEE (2007). 
Las áreas del conocimiento en las que tienen formación los presidentes electos son: en primer lugar educación; en segundo, agronomía; y en tercer lugar, administración de empresas. Se tiene otras áreas como geoquímica, mecánica, turismo, contaduría pública, petróleo y medicina.

Tomando en consideración las tres fuerzas políticas de México: PAN, PRI y PRD, en este proceso electoral aparece el PAN con 28 presidencias municipales (en este caso hizo alianzas con los partidos PCP y PNA); el PRI con 23 (hizo alianza con el PASC y con PCP); y el PRD con 4 (quien realizó alianza con el PT y PC).

\section{Perfil profesional de los actores electos en el proceso político 2009}

Pasando a los comicios electorales del 2009, se tuvieron los resultados que se presentan en los siguientes renglones.

\section{- Diputados locales de mayoría relativa}

Al igual que las elecciones del 2006, de los 15 diputados electos, nuevamente el 86.66 son del sexo masculino y $13.33 \%$ del femenino. El $60 \%$ de los diputados cuentan con el nivel de licenciatura / ingeniería, aunque dos de ellos cuentan con el grado de maestría. El 26.66\% cuentan con preparatoria, mientras que el $13.33 \%$ solamente tienen estudios de secundaria. Las áreas del conocimiento más representativas en cuanto a la formación profesional de estos servidores públicos son (con igual número de actores): ciencias políticas, derecho y contaduría.

Por lo que se refiere a los partidos políticos postulantes de los diputados, tenemos que el PAN cuenta con 9 diputados (en alianza con el PNA). El PRI cuenta con seis posiciones (en alianza con el PVEM y el PSD).

\section{- Diputados locales de representación proporcional}


En el caso de los 12 diputados electos, el $66.66 \%$ son del género masculino y el $33.33 \%$ del femenino. En lo que se refiere al nivel académico, el $58.33 \%$ cuentan con el grado de licenciatura / ingeniería; el 25\% tienen maestría; uno de ellos solamente cursó preparatoria y otro cuenta con secundaria.

Las áreas de conocimiento que predominan en la formación profesional de los diputados son: en primer lugar, derecho; y le siguen con igual proporción, administración, contaduría y educación. Los diputados electos pertenecen a los siguientes partidos políticos: tres al PAN, tres al PRI, uno al PRD, uno al PT, uno al PVEM y uno al PCP.

\section{- Presidentes municipales}

En el proceso electoral que se analiza, se eligieron 58 presidentes municipales. Al igual que en el proceso electoral del 2006, de igual manera llama la atención que de los actores políticos electos, el $91.37 \%$ son del sexo masculino (53), contra un $8.62 \%$ que pertenecen al sexo femenino (5). La formación académica de los servidores electos se muestra en la Tabla 9.

Tabla 9. Nivel académico de los presidentes municipales electos en el proceso del 2009 en el Estado de SLP.

\begin{tabular}{|l|c|c|}
\hline \multicolumn{1}{|c|}{ Nivel académico concluido } & Cantidad & $\begin{array}{c}\text { Porcentaje del total de presidentes } \\
\text { municipales electos }\end{array}$ \\
\hline Maestría & 2 & $3.44 \%$ \\
\hline Licenciatura / ingeniería & 27 & $46.55 \%$ \\
\hline $\begin{array}{l}\text { Bachillerato / preparatoria / carrera } \\
\text { técnica }\end{array}$ & 13 & $22.41 \%$ \\
\hline Secundaria & 8 & $13.79 \%$ \\
\hline Primaria & 6 & $10.34 \%$ \\
\hline Primaria inconclusa (4 ${ }^{\circ}$ grado) & 2 & $3.44 \%$ \\
\hline
\end{tabular}

Fuente: Elaborada por el autor a partir de CEEPAC (2009). 
Las áreas del conocimiento en las que tienen formación los presidentes electos son: en primer lugar derecho; en segundo, educación / pedagogía; en tercer lugar, contaduría, medicina y agronomía; y en cuarto lugar con la misma cantidad ingeniería urbana, edificación de obras y medicina. Se tiene otras áreas como psicología, mecánica, y gastronomía.

Tomando en consideración las tres fuerzas políticas de México: PAN, PRI y PRD, en este proceso electoral aparece el PAN con 24 presidencias municipales (en este caso hizo alianzas con los partidos PCP, PNA y PT); el PRI con 21 (hizo alianza con el PSD, PVEM y PNA); y el PRD con 5 (quien realizó alianza con el PT, PCP y el PSD). El resto de las presidencias municipales quedaron entre otros partidos nacionales y locales.

\section{Perfil profesional de los actores electos en el proceso político 2012}

Siguiendo con los comicios electorales del 2012, se tuvieron los resultados que se presentan en los siguientes renglones.

\section{- Diputados locales de mayoría relativa}

Por lo que hace a los resultados del proceso electoral 2012, de los 15 diputados electos, el $80 \%$ son del sexo masculino y el $20 \%$ del femenino. El $33.33 \%$ de los diputados cuentan con el nivel de licenciatura / ingeniería, mientras que el $26.66 \%$, cuentan con el grado de maestría. El $26.66 \%$ cuentan con preparatoria o carrera técnica, mientras que el $6.66 \%$ solamente tienen estudios de secundaria. Las áreas del conocimiento de las licenciaturas de los actores revisados son multidisciplinarias, por ejemplo: derecho, ciencias sociales, agronomía, arquitectura, contaduría pública e ingeniería mecánica. En el caso de los actores que cuentan con maestría, ésta es en las áreas de mercadotecnia, gestión pública, salud pública y educación. 
Por lo que se refiere a los partidos políticos postulantes de los diputados, tenemos que el PRI cuenta con 1 diputado y 9 en alianza con el PVEM. El PAN en alianza con el PNA cuenta con 4 posiciones y el PRD en alianza con el PT, tiene una diputación.

\section{- Diputados locales de representación proporcional}

En el caso de los 12 diputados electos, el $83.33 \%$ son del género masculino y el $16.67 \%$ del femenino. En lo que se refiere al nivel académico, el 75\% cuentan con el grado de licenciatura / ingeniería; el $16.67 \%$ tienen maestría; mientras que el $8.33 \%$ cuentan con preparatoria o carrera técnica.

Las áreas de conocimiento que predominan en la formación profesional de los diputados son áreas multidisciplinarias: arquitectura, contaduría pública, derecho, pedagogía, enfermería, ingeniería geoquímica, educación secundaria y administración de empresas. Los diputados electos pertenecen a los siguientes partidos políticos: dos al PAN, dos al PRI, dos al PVEM, dos al PNA, uno al PRD, uno al PT, uno al PCP y uno al PMC.

\section{- Presidentes municipales}

En el proceso electoral que se analiza, se eligieron 58 presidentes municipales. Del total de presidentes municipales electos, el $89.66 \%$ son del sexo masculino (52), y solamente el $10.34 \%$ pertenecen al sexo femenino (6). La formación académica de los servidores electos se muestra en la Tabla 10. 
Vol. 6, No. 11, 2016.

Tabla 10. Nivel académico de los presidentes municipales electos en el proceso del 2012 en el Estado de SLP.

\begin{tabular}{|l|c|c|}
\hline \multicolumn{1}{|c|}{ Nivel académico concluido } & Cantidad & $\begin{array}{c}\text { Porcentaje del total de } \\
\text { presidentes municipales electos }\end{array}$ \\
\hline Maestría & 4 & $6.89 \%$ \\
\hline Licenciatura / ingeniería & 31 & $53.44 \%$ \\
\hline $\begin{array}{l}\text { Bachillerato / preparatoria / carrera } \\
\text { técnica }\end{array}$ & 10 & $17.24 \%$ \\
\hline Secundaria & 8 & $13.80 \%$ \\
\hline Primaria & 5 & $8.63 \%$ \\
\hline
\end{tabular}

Fuente: Elaborada por el autor a partir de CEEPAC (2013).

Las áreas del conocimiento en las que tienen formación los presidentes electos son: en primer lugar educación / pedagogía; en segundo lugar, derecho; en tercer lugar, agronomía; y en cuarto lugar con la misma cantidad, contaduría pública y administración de empresas. Se tiene otras áreas como ingeniería química, ingeniería en topografía, ciencias sociales y ciencias de la comunicación.

En el proceso electoral analizado, el PRI en alianza con el PVEM obtuvo 31 presidencias municipales, 2 de manera individual, una en alianza con el PCP, y otra en alianza con el PVEM y el PCP. Por otro lado, el PAN de manera individual obtuvo una presidencia municipal, 9 en alianza con el PNA y otra en alianza con los partidos políticos PRD, PT, PVEM, PCP, PMC y PNA. El PT obtuvo 2 presidencias municipales de manera individual, una en alianza con el PMC, 3 en alianza con el PRD y el PMC, además de la obtenida en alianza con el PAN, PRD, PVEM, PCP, PMC y PNA. El PCP obtuvo 4 presidencias de forma individual, una en alianza con el PRI, una en alianza con el PRD, una en alianza con el PRI y el PVEM, y una en alianza con el PAN, PRD, PT, PVEM, PMC y PNA. Finalmente, el PNA obtuvo una presidencia municipal de manera individual, 9 en alianza con el PAN, y una en alianza con el PAN, PRD, PT, PVEM, PCP y PMC. 


\section{A manera de conclusión}

De la revisión y análisis de los datos utilizados para la construcción de este trabajo, se puede concluir que el perfil profesional de los actores políticos -al menos en cuanto a grado académico- sigue siendo bajo. No obstante que se percibe un mejoramiento en cuanto a la preparación académica, ésta tiene poca -o nula en una buena parte de los casos- relación con el ámbito de la gestión pública, hablando tanto de los cargos de diputado como de los de presidente municipal.

La participación de la mujer sigue siendo baja en este tipo de procesos políticos, por lo que al menos para el caso de San Luis Potosí- la clase política local es eminentemente del género masculino. Vale la pena señalar, que sería pertinente buscar a través de otros estudios, cuál es la razón o razones que provocan esta cuestión.

También se puede comentar que los esquemas que utilizan los diferentes partidos políticos tanto nacionales como locales- siguen siendo deficientes e insuficientes para allegar los mejores elementos humanos que la administración pública requiere para hacer más efectivo su desempeño, al tratar de satisfacer las demandas de la ciudadanía en general.

De acuerdo a los datos biográficos revisados para este trabajo, se observa que no prevalece un partido sobre otro, tratando de postular a los mejores hombres y mujeres hacia la vida política; es decir, el perfil profesional de los candidatos es muy similar entre todas las fuerzas políticas. En este caso, también se recomienda que a través de otros trabajos se indague sobre cuál es el mecanismo o mecanismos que los diferentes partidos políticos utilizan para elegir a sus candidatos, por los que la ciudadanía invariablemente tiene que votar, aun cuando éstos no sean del agrado total de los votantes. 
De acuerdo con los datos revisados de los tres procesos políticos, se observa que al menos para el ámbito local de San Luis Potosí, los partidos políticos han implantado el sistema también conocido como partidocracia. Ningún partido político hasta la fecha, ha mostrado señales evidentes de buscar otros esquemas para hacer política, con el propósito de profesionalizar la administración pública. Es como dice Sartori (Arce, 2006), los partidos políticos se han degenerado en grupos de facción. En este mismo tenor de ideas, se menciona en un estudio que realizó la OCDE sobre la gestión pública en México (OCDE, 2011), que “el sistema de apropiación partidista de los cargos públicos que ha permeado las prácticas de recursos humanos en el país ha sido un obstáculo para la diversidad, ya que los diversos grupos -las camarillas- que llegan a la administración pública están formados por personas que normalmente poseen los mismos antecedentes académicos, económicos y sociales”.

De hecho, apoyándonos en los comentarios vertidos en el punto inmediato que antecede, en el caso de los procesos electorales celebrados en el Estado de San Luis Potosí en 2006, 2009 y 2012, se presentan resultados muy similares -no dudamos que esta situación prevalezca en el caso de los procesos electorales subsecuentes-. Entre los resultados parecidos que se encontraron, se tiene que: la cuota de género es igual en los tres procesos, el nivel de escolaridad de los candidatos se mantiene casi igual, el área del conocimiento en la que están formados dichos actores políticos es muy semejante, el comportamiento de todos los partidos políticos participantes en los referidos comicios electorales es igual -no destaca un partido político sobre otro, que intente cambiar la situación-.

Es de llamar la atención que el comportamiento de los votantes es parecido en casi todos los casos, ya que se aprecia que de un proceso a otro, van cambiando de partido tratando de buscar alguna opción mejor, pero al final sucede lo mismo con el desempeño de los políticos en el poder. En los datos revisados, se pudo observar también que los actores políticos van 
cambiando de un puesto a otro, como si en verdad contaran con el mejor perfil profesional requerido para el desempeño de la encomienda asignada. 


\section{Referencias}

Arce T., C. A. "La gobernabilidad democrática: una perspectiva para el desarrollo". México, Editado por la H. Cámara de Diputados (LIX Legislatura) y Miguel Ángel Porrúa, primera edición. (2006).

Blacha, L. E. ¿Élite o clase política? algunas precisiones terminológicas. Revista Theomai, núm. $12 . \quad$ (2005). Recuperada de http://www.redalyc.org/articulo.oa?id=12401210

Consejo Estatal Electoral de San Luis Potosí [CEE]. “Los electos”. México, CEEPAC, primera edición. (2007).

Consejo Estatal Electoral y de Participación Ciudadana de San Luis Potosí [CEEPAC]. Los electos: proceso electoral 2009. México, CEEPAC, primera edición. (2009).

Consejo Estatal Electoral y de Participación Ciudadana de San Luis Potosí [CEEPAC]. Los electos: proceso electoral 2012. México, CEEPAC, primera edición. (2013).

Hurtado, J. La clase política jalisciense: 1947-1992. Espiral, II() 105-134. (1996). Recuperado de http://www.redalyc.org/articulo.oa?id=13820505

Mosca, G. La clase política1. (1972). Recuperado de http://uenf.br/cch/lesce/files/2013/03/Mosca-cap-II-em-português.pdf

Organización para la Cooperación y el Desarrollo Económicos [OCDE]. "Hacia una gestión pública más efectiva y dinámica”. México, editado por la OCDE (Estudios de la OCDE sobre gobernanza pública), primera edición. (2011).

Pérez G., H. N. "Retos y perspectivas del servicio público de carrera en México". En, Servicio profesional de carrera. México, editado por Red Mexicana de Servicio Profesional, A. C. y Fundación Mexicana de Estudios Políticos y Administrativos, A. C., Volumen 1, núm. 1. (2004).

Vega C., M. A. "Racionalidad administrativa y racionalidad política en el reposicionamiento de actores gubernamentales: el caso de San Luis Potosí 1979. 2004”. México, Universidad Autónoma de San Luis Potosí, Tesis Doctoral. (2008).

Vega C., M. A. El perfil profesional de la democracia local: servidores públicos electos en los procesos políticos 2006 y 2009 en San Luis Potosí, México. En Santillán C., F y Martínez I., J. E. "Gestión de proyectos de investigación enfocados a la educación y sociedad". México: Editorial Cenid, Primera edición, pp. 113-134. (2016).

\section{Documentos legales}

Constitución Política de los Estados Unidos Mexicanos. Documento obtenido de: http://www.diputados.gob.mx/LeyesBiblio/pdf/1.pdf Fecha de consulta: 10/08/2013. 
Constitución Política del Estado Libre y Soberano de San Luis Potosí. Documento obtenido de

http://148.235.65.21/sites/default/files/unpload/legislacion/leyes/2015/12/Constituci ón\%2 0Pol\%C3\%ADtica\%20del\%20Estado.pdf Fecha de consulta: 29/05/2016.

Ley orgánica del Municipio Libre de San Luis Potosí. Documento obtenido de http://148.235.65.21/sites/default/files/unpload/legislacion/leyes/2016/05/99_Ley_O rgani ca_del_Municipio_Libre_del_Estado.pdf Fecha de consulta: 29/05/2016. 